\title{
Yield Loss Due to Septoria tritici Blotch (Septoria Tritici) of Bread Wheat (Triticum aestivum L.) in the Central Highlands of Ethiopia
}

\author{
Yitagesu Tadesse ${ }^{1,2, *} \quad$ Alemayehu Chala ${ }^{1} \quad$ Bekele Kassa $^{2}$ \\ 1.College of Agriculture, Hawassa University, Hawassa, Ethiopia \\ 2.Ethiopian Institute of Agricultural Research, Holleta Agricultural Research Center, Addis Ababa, Ethiopia
}

\begin{abstract}
Septoria tritici blotch (STB) is an economically important foliar disease on wheat. The current research was conducted to determine the yield loss of STB on wheat at Holeta and Kulumsa in 2017. Maximum relative grain yield loss $(36 \%)$ was recorded on unsprayed plots of the variety Kekeba at Holetta. Whereas; at Kulumsa, maximum relative grain yield loss (39\%) was on unsprayed plots of the variety Madawalabu. Disease severity, incidence and AUDPC showed highly significant and negative correlations with grain yield, spike length, plant height, and weight of kernel per spike, kernel number per spike, thousand seed weight and hectoliter weight. The present findings confirmed the importance of STB in Ethiopia and the role fungicides play in managing the disease on partially resistant varieties.
\end{abstract}

Keywords: Correlation, Septoria tritici blotch, Yield Loss, Yield recovery.

DOI: $10.7176 / \mathrm{JBAH} / 10-10-01$

Publication date:May $31^{\text {st }} 2020$

\section{Background and Justification}

Wheat is one of the oldest cereal crops. It is grown under a wide range of cli-mates and soils and adapted to temperate regions with annual rainfall of $30-90 \mathrm{~cm}(\mathrm{FAO}, 2017)$. It is the fourth most important cereal crop in agriculture after teff (Eragrostis tef (Zucc.)Trotter), maize (Zea mays L) and sorghum (Sorghum bicolor (L.) Moench). In terms of total grain production, it ranks third after maize and teff (CSA, 2017). The crop is widely cultivated at altitudes ranging from 1500 to 3000 m.a.s.l, in Ethiopia, the most suitable area falls between 1700 and 2800 m.a.s.l. Ethiopia is the second largest producer of wheat in Sub-Saharan Africa after South Africa (Negasa and Chauhin, 2016). In spite of the production and yield increases, average grain yield of wheat is still low $(<2.7 \mathrm{t} / \mathrm{ha})$ and highly variable and below the world's average (3.09 t/ha) $(\mathrm{FAO}, 2017)$.

The crop can be grown in most locations where annual rainfall ranges from 250 to $1750 \mathrm{~mm}$. About $75 \%$ of the wheat grown world-wide receives an average rainfall between 375 and $875 \mathrm{~mm}$ annually. However, too much precipitation can lead to yield loss from diseases and poor root growth and development problems (Blandford, D. and Hassapoyannes, K., 2018).

Despite its importance as food and industrial crop, wheat production and productivity around the globe is hampered by a number of factors including biotic and abiotic stresses as well as low adoption of new agricultural technologies (Bharadwaj, D.N., 2016). STB caused by the fungus Septoria tritici (Mycosphaerella graminicola), is a major disease of wheat in all wheat-growing areas of the world causing serious economic losses (Singh et al., 2016). It is one of the most aggressive diseases on common wheat (Triticum aestivum L.) and durum wheat ( $T$. turgidum L. var. durum) globally (Serfling et al., 2017). Septoria tritici blotch is by far the most important disease in Northern and Eastern Africa and the Middle East (Benbelkacem, 2016). However, according to Tadesse et al. (2018), the prevalence and severity of the disease is more dependent on weather conditions of the season and varieties grown. The disease is one of the major constraints of wheat in all wheat-growing areas of Ethiopia, causing $42 \%$ economic loss annually (Takele et al., 2015; Said et al., 2016).

Range of disease management options are recommended to control STB in wheat fields. Among these, cultural management options designed to reduce inoculum pressure are the first one. Bio-control has also been tested as another STB management option. Pseudomonads have been tested as potential biocontrol agents (Ponomarkeno et al., 2011). Fungicides of various modes of actions have been recommended to manage STB but their use in Ethiopia has been limited mainly due to economic reasons. Breeding for resistance is likely to be the most practical method of controlling STB (Takele et al., 2015). Several sources of resistance have been reported but breeding for resistance has not always been successful in protecting wheat from the damaging effects of the disease; as expression of resistance is often correlated with morphological traits. Moreover, wheat cultivars resistant in one part of the world may display susceptibility elsewhere. Even within a country, a difference observed in pathogen virulence that may be associated with fungal genetic variability (McDonald et al., 2016) is hindering the development of wheat varieties with broad spectrum of resistance. Selection for partial resistance to STB may be restricted if that trait has a significant cost, for example reduced yield, which is the most important target for many wheat breeders (Miedaner et al., 2016). 
Overall STB has remained an important constraint to wheat production all over the world including in Ethiopia (Randhawa et al., 2019). However, effective and sustainable managing of the disease is yet to be achieved under Ethiopian condition. Septoria tritici blotch (STB) is one of the major diseases of wheat around the world and across wheat growing regions of Ethiopia. Yield loss assessment studies have been carried out in fewer areas and they are largely based on data from field surveys. As a result there it is very crucial to develop disease management option and recommended in areas, where the disease is prevalent and economically important. Thus, our study was designed with the objectives of determine the yield loss of each cultivars due to STB and determine the correlation between yield components and disease parameters.

\section{Materials and Methods}

\section{Study areas}

Our study conducted at Holetta and Kulumsa Agricultural Research Centers, Ethiopia. Both sites are suitable for bread wheat production, and STB pressure is generally high during the rainy season (Birhan, 2011; Beyene et al., 2016).

\section{Treatments and Experimental Design}

The treatment arrangement of our experiment was ( 6 different spray schedules of propiconazole (Tilt 250 EC) and Mancozeb (unsprayed plot, Tilt, Mancozeb, Mancozeb-Tilt-mancozeb-Tilt, Tilt-mancozeb- Tilt-mancozeb and mancozeb- mancozeb- Tilt- Tilt) were combined with three wheat varieties (Alidoro, Kekeba and Madawalabu). Our treatments were arranged in randomized complete block design (RCBD) with three replications. The fungicide Tilt was applied at a rate of $0.5 \mathrm{lt} / \mathrm{ha}$ and Mancozeb at a rate of $3 \mathrm{~kg} / \mathrm{ha}$ based on recommendation.

\section{Data collected}

Incidence of STB was assessed by counting the number of infected plants in the middle four rows and was expressed as percentage of total plants infected as shown below.

$$
\text { Disease Incidence }=\text { No. of disease } \frac{\text { plants }}{\text { Total }} \text { no. of plants } * 100
$$

The severity of Septoria tritici blotch was recorded using the double-digit scale (00-99) (Saari and Prescott, 1975; Eyal et al., 1987). Percent disease severity is estimated based on the formula:

$$
\% \text { Disease Severity }(P D S)=\left(\frac{D 1}{Y 1}\right) *\left(\frac{D 2}{Y 2}\right) * 100
$$

Where D1 and D2 represent the score recorded (00-99 scale) and Y1 and Y2 represent the maximum score on the scale (9 and 9) (Sharma and Duveiller, 2007).

$$
\text { AUDPC }=\sum_{\mathbf{i}=\mathbf{1}}^{\mathrm{Ni}-\mathbf{1}} \frac{(\mathbf{X i}+\mathbf{X} \mathbf{i}+\mathbf{1})}{2}(\mathbf{t i}+\mathbf{1}-\mathbf{t i}) \text { Where, }
$$

$\mathrm{Xi}=$ the cumulative disease severity expressed as a proportion at the $\mathrm{i}^{\text {th }}$ observation,

$\mathrm{ti}=$ the time (days after planting) at the $\mathrm{i}^{\text {th }}$ observation and

$\mathrm{n}=$ total number of observations. AUDPC values are used in analysis of variance to compare amount of disease among different treatments. Relative losses in yield and yield component of each variety were determined as a percentage of that of the protected plots of the respective variety.

Losses were calculated separately for each of the treatments as:

$$
R L(\%)=\frac{(Y 1-Y 2)}{Y 1} * 100 \%
$$

Where, RL - relative loss (reduction of the parameters yield, yield component), Y1 - mean of the respective parameter on protected plots (plots with maximum protection) and Y2 - mean of the respective parameter in unprotected plots (i.e. unsprayed plots or sprayed plots with varying level of disease). Percent yield recovery was also calculated to compare the yield difference among fungicide treatments using the formula:

$$
\boldsymbol{Y R}(\%)=\boldsymbol{Y S P}-\frac{\boldsymbol{Y U P}}{\boldsymbol{Y U P}} * \mathbf{1 0 0} \%
$$

Where, YR is yield recovery in percent, YUP is yield of unsprayed plot and YSP is yield of sprayed plots. All agronomic, yield and yield related data were recorded on the middle four rows of each experimental plot.

\section{Data Analysis}

Data analysis was carried out by SAS computer package version 9.3 (SAS, 2014). Means for treatments were compared using Duncan's New Multiple Range Test. Correlation analysis was done using the Proc Corr procedures to see the relationship between yield, yield components and disease parameters. 


\section{Results and Discussion}

Disease incidence in percent

STB incidences were significantly different among varieties at both locations. The highest disease incidence was recorded on unsprayed plots of Kekeba variety, while the lowest disease incidence was recorded on Alidoro variety sprayed with Tilt fungicide (Table 1). This result strongly agreed with the finding of (Said et al., 2016), who reported maximum STB incidence value from unsprayed plots of each varieties.

Table 1. Cummulative impact of wheat cultivars and Mode of application of chemicals.

\begin{tabular}{|c|c|c|c|c|c|c|c|}
\hline \multirow[b]{2}{*}{ Wheat cultivars } & \multirow[b]{2}{*}{ Schedule } & \multicolumn{3}{|l|}{ Holetta } & \multicolumn{3}{|l|}{ Kulumsa } \\
\hline & & Final Incide & Final Severity & AUDPC & Final Incide & Final Severity & AUDPC \\
\hline \multirow{6}{*}{ Alidoro } & Control & $50^{\mathrm{abc}}$ & $85^{\text {abcd }}$ & $1733.7^{\mathrm{de}}$ & $10^{\mathrm{cd}}$ & $35.3^{\mathrm{def}}$ & $761.8^{\mathrm{d}}$ \\
\hline & Mancozeb & $21.7^{\mathrm{f}}$ & $59.3^{\text {efg }}$ & $1041.8^{\mathrm{ef}}$ & $6.67^{\mathrm{d}}$ & $28.7^{\mathrm{ef}}$ & $677.8^{\mathrm{d}}$ \\
\hline & Tilt & $16.7^{\mathrm{fg}}$ & $45.3^{\mathrm{g}}$ & $992.8^{\mathrm{ef}}$ & $8.33^{\mathrm{cd}}$ & $21.7^{\mathrm{f}}$ & $634.7^{\mathrm{d}}$ \\
\hline & MMTT & $21.7^{\mathrm{f}}$ & $56.7^{\mathrm{efg}}$ & $1086.2^{\mathrm{ef}}$ & $8.33^{\mathrm{cd}}$ & $22.3^{\mathrm{f}}$ & $593.8^{d}$ \\
\hline & TTMM & $21.7^{\mathrm{f}}$ & $56^{\mathrm{efg}}$ & $1037.2^{\text {ef }}$ & $5^{\mathrm{d}}$ & $21.3^{\mathrm{f}}$ & $592.7^{\mathrm{d}}$ \\
\hline & MTMT & $10^{\mathrm{g}}$ & $49.7^{\mathrm{fg}}$ & $865.7^{\mathrm{f}}$ & $6.67^{\mathrm{d}}$ & $22.3^{\mathrm{f}}$ & $591.5^{\mathrm{d}}$ \\
\hline \multirow{7}{*}{ Kekeba } & Control & $98.3^{\mathrm{a}}$ & $96.7^{\mathrm{a}}$ & $3879.2^{\mathrm{a}}$ & $66.67^{\mathrm{a}}$ & $62.7^{\mathrm{b}}$ & $1698.7^{b}$ \\
\hline & Mancozeb & $66.7^{\mathrm{bcd}}$ & $78.3^{\text {abcde }}$ & $2146.7^{\mathrm{cd}}$ & $30^{\mathrm{bcd}}$ & $49^{\text {bcd }}$ & $1403.5^{c}$ \\
\hline & Tilt & $53.3^{\mathrm{abc}}$ & $70.3^{\text {bcdef }}$ & $2240^{\mathrm{bcd}}$ & $35^{\mathrm{bc}}$ & $46^{\mathrm{cd}}$ & $1295^{\mathrm{c}}$ \\
\hline & MMTT & $71.7^{\mathrm{bc}}$ & $88^{\mathrm{abc}}$ & $2952.8^{b}$ & $48.33^{\mathrm{ab}}$ & $52^{\mathrm{bc}}$ & $1410.5^{c}$ \\
\hline & TTMM & $63.3^{\mathrm{bcd}}$ & $73.3^{\text {bcde }}$ & $2173.5^{\mathrm{cd}}$ & $26.67^{\mathrm{bcd}}$ & $38.7^{\mathrm{cde}}$ & $1289.2^{\mathrm{c}}$ \\
\hline & MTMT & $45^{\text {cdef }}$ & $62.3^{\operatorname{defg}}$ & $1894.7^{\mathrm{d}}$ & $16.67^{\mathrm{cd}}$ & $48^{\mathrm{bcd}}$ & $1422.2^{\mathrm{c}}$ \\
\hline & Control & $83.3^{\mathrm{ab}}$ & $92.7^{\mathrm{ab}}$ & $2889.8^{\mathrm{bc}}$ & $31.67^{\mathrm{bcd}}$ & $84.7^{\mathrm{a}}$ & $2056.8^{a}$ \\
\hline \multirow{7}{*}{ Madawalabu } & Mancozeb & $60^{\mathrm{bcd}}$ & $85^{\mathrm{abcd}}$ & $2121^{\mathrm{cd}}$ & $21.67^{\mathrm{cd}}$ & $62^{b}$ & $1639.2^{b}$ \\
\hline & Tilt & $38.3^{\mathrm{def}}$ & $69^{\text {cdef }}$ & $1456^{\text {def }}$ & $10^{\mathrm{cd}}$ & $39^{\text {cde }}$ & $1272.8^{c}$ \\
\hline & MMTT & $45^{\text {cdef }}$ & $69.7^{\text {cdef }}$ & $1799^{\text {de }}$ & $13.33^{\mathrm{cd}}$ & $49^{\text {bcd }}$ & $1382.5^{\mathrm{c}}$ \\
\hline & TTMM & $38.3^{\mathrm{def}}$ & $69^{\text {cdef }}$ & $1488.7^{\mathrm{def}}$ & $13.33^{\mathrm{cd}}$ & $42^{\text {cde }}$ & $1331.2^{\mathrm{c}}$ \\
\hline & MTMT & $30^{\mathrm{ef}}$ & $59.3^{\text {efg }}$ & $1457.2^{\text {def }}$ & $15^{\mathrm{cd}}$ & $46.3^{\mathrm{cd}}$ & $1369.7^{\mathrm{c}}$ \\
\hline & Mean & 46.4 & 70.3 & 1847.55 & 20.74 & 42.8 & 1190.2 \\
\hline & $\mathrm{CV}$ & 23.4 & 9.3 & 23.04 & 32.05 & 8.9 & 10.88 \\
\hline
\end{tabular}

$\mathrm{CV}=$ Coefficient of Variation, MMTT=Mancozeb-Mancozeb-Tilt-Tilt, TTMM=Tilt-Tilt-Mancozeb-Mancozeb, MTMT= Mancozeb-Tilt - Mancozeb-Tilt

\section{Percent disease severity}

At Holeta the highest (97\%) severity was recorded on unsprayed plots of variety Kekeba. While the lowest (45\%) disease severity was recorded from Alidoro variety sprayed with Tilt. At Kulumsa the highest (85\%) disease severity was recorded from Madawalabu variety unsprayed plots, while the lowest $(22 \%)$ disease severity was recorded from Alidoro variety sprayed with different fungicides. This indicates that the level of disease development is considerably affected by level of fungicide application or varietal resistance to STB.

In general, STB was severe in both locations; however, it was more severe at Holeta than at Kulumsa. According to results of the present study, the currently grown high yielding wheat variety, Kekeba, was the most susceptible to STB suggesting the need to prioritize the deployment of resistance genes. Use of resistant variety is the best control strategy to Septoria tritici blotch for resource poor farmers in developing countries and the most environmentally friendly and profitable strategy for commercial farmers (Tekelay et al., 2015).

\section{Area under disease progress curve}

STB AUDPC values across treatments ranged from 866 to 3879 at Holeta and from 592 to 2057 at Kulumsa (Table1). AUDPCs were generally higher on unsprayed plots than on sprayed plots. The maximum AUDPC values recorded on unsprayed plots were 3879\%-days on wheat variety Kekeba, $2890 \%$-days on Madawalabu and 1734\%-days on Alidoro, at Holeta respectively. At Kulumsa, AUDPC values recorded as follows: 2057\%-days, 1699\%-days and $762 \%$-days, on Madawalabu, Kekeba and Alidoro varieties, respectively. Wheat variety Alidoro sprayed with MTMT fungicides schedules had the lowest (866\%-days) at Holeta and variety Alidoro treated with MTMT fungicide had the lowest AUDPC (591\%-days) at Kulumsa. All fungicide spray schedules have reduced AUDPC compared to the unsprayed plots. This agrees with that of Takele et al., (2015) who reported maximum AUDPC values (2275\%-days) from unsprayed plots.

\section{Yield and Yield Components Loss}

Yield loss

At Holeta, maximum relative grain yield loss was $36 \%$, and this was recorded on unsprayed plots of the variety 
Kekeba (Table 2). On Madawalabu variety, grain yield loss of about 34\% was recorded when STB was allowed to develop naturally. For Alidoro variety, grain yield reduction of about $35 \%$ was recorded on unsprayed plots.

At Kulumsa, maximum relative grain yield loss was 39\% on unsprayed plots of the variety Madawalabu. On the variety Kekeba, grain yield loss of about $16.1 \%$ was recorded from unprotected plots, while grain yield reduction of about $21 \%$ was recorded on unsprayed plots of the variety Alidoro (Table 2 ).

Our result agrees with the findings of Said et al. (2016) in which STB was reported causing grain yield loss of up to $41 \%$. It confirmed that susceptible genotypes showed higher yield losses as compared to resistant genotypes. Our results also confirm the findings of Goodwin et al. (2011) in which wheat grain yield losses of 30$50 \%$ were attributed to STB. Severe yield losses can occur in crops when the top two or three leaves (flag, second and third leaf of wheat plants) become infected (Ayele et al., 2019).

\section{Loss in thousand kernel weight}

At Holeta, thousand kernel weight (TKW) was most affected on wheat variety Kekeba. The loss in TKW on this variety reached $25 \%$ on unsprayed plots (Table 2 ). On the other hand, a $7 \%$ loss in TKW was recorded on unsprayed plots of variety Alidoro, which is considered the lowest loss in TKW.

At Kulumsa, thousand kernel weight (TKW) was most affected on wheat variety Kekeba. The loss in TKW on this variety reached $18 \%$ on unsprayed plots (Table 2 ). On the other hand, $6.8 \%$ loss in TKW was recorded on unsprayed plots of variety Alidoro, which is considered as the lowest loss in TKW. Current results are in agreement with previous reports of Takele et al. (2015) in which yield, thousand seed weight, hectoliter weight, weight of kernel per spike, losses, due to STB were recorded as $41,36,5$ and $44 \%$, respectively. The reduction in TKW is mainly due to the effect of the disease on the size and mass of the seed (shriveling of the kernels). Dill-Macky et al. (1990) reported up to $45 \%$ reduction in 1000-kernel weight of wheat variety Oxley in Australia. Infection of wheat head and leaf sheaths by septoria affects the transport of assimilates to the developing kernel and results in shriveled kernel (Sharma et al., 2002).

\section{Hectoliter weight loss}

Only the Tilt fungicide treatments showed significant effect $(\mathrm{P}<0.05)$ over the control in terms of hectoliter weight of the variety Kekeba and Madawalabu. None of the spray treatments showed significant effect on hectoliter weight of Alidoro (Table 2). Reduction in test (hectoliter) weight is largely attributed to reduction in seed size. With small size more seeds would fit into a given volume. Even though not quantified, Ayele et al. (2019) reported pronounced effect of septoria on hectoliter weight and kernel plumpness of some winter Australian wheat cultivars. However, the effect may vary depending on the variety and location (Dill-Macky et al., 1990).

Table 2. Effect of fungicide Spray Schedules on yield and yield components loss $(\%)$.

\begin{tabular}{|c|c|c|c|c|c|c|c|c|c|c|c|c|c|}
\hline \multicolumn{2}{|c|}{ Treatments } & \multicolumn{6}{|c|}{ Holetta } & \multirow[b]{2}{*}{ TKW } & \multirow[b]{2}{*}{ RTL } & \multicolumn{4}{|c|}{ Kulumsa } \\
\hline \multirow[t]{2}{*}{ Variety } & Fungicide & TKW & RTL & HLW & RHL & Yield & RYL & & & HLW & RHL & Yield & RYL \\
\hline & Control & $39.2^{\mathrm{fg}}$ & 7.3 & $74.6^{\mathrm{ab}}$ & 2.9 & $3.2^{\mathrm{de}}$ & 35 & $37^{\text {defg }}$ & 6.8 & $78.5^{\mathrm{bcd}}$ & 1.1 & $3.7^{\mathrm{ab}}$ & 20.8 \\
\hline \multirow[t]{7}{*}{ Alidoro } & Mancozeb & $42.3^{\text {bcdef }}$ & 0 & $76^{\mathrm{ab}}$ & 1.1 & $4.2^{\mathrm{abc}}$ & 15 & $39^{\text {abcde }}$ & 0 & $78.8^{\mathrm{bcd}}$ & 0.7 & $3.95^{\mathrm{ab}}$ & 16.14 \\
\hline & Tilt & $41.3^{\text {cdef }}$ & 2.4 & $76.9^{\mathrm{a}}$ & 0 & $4.9^{\mathrm{abcd}}$ & 0 & $39^{\text {abcdefg }}$ & 0.8 & $79^{\mathrm{abcd}}$ & 0.2 & $4.7^{\mathrm{a}}$ & 0 \\
\hline & MMTT & $40.27^{\mathrm{efg}}$ & 4.7 & $76.4^{\mathrm{ab}}$ & 0.6 & $4.3^{\mathrm{abc}}$ & 13 & $38.7^{\text {abcdefg }}$ & 1.7 & $79.3^{\mathrm{abc}}$ & 0 & $4.38^{\mathrm{a}}$ & 7.01 \\
\hline & TTMM & $40.8^{\operatorname{defg}}$ & 3.5 & $76.9^{\mathrm{a}}$ & 0 & $4.5^{\mathrm{ab}}$ & 8.5 & $37.1^{\text {bcdefg }}$ & 5.8 & $78.9^{\mathrm{bcd}}$ & 0.6 & $4.6^{\mathrm{a}}$ & 2.34 \\
\hline & MTMT & $40.4^{\text {efg }}$ & 4.4 & $75.3^{\mathrm{ab}}$ & 2.1 & $4.3^{\mathrm{abc}}$ & 13 & & 27 & & 0.5 & $455^{\mathrm{a}}$ & 3.4 \\
\hline & & & & & & & & $38^{\text {abcdefg }}$ & 2.1 & $79^{\text {bed }}$ & & 4.55 & \\
\hline & Control & $35.2^{\mathrm{h}}$ & 25 & $72.7^{\mathrm{b}}$ & 5.2 & $3.3^{\mathrm{de}}$ & 36 & $34.7^{\mathrm{g}}$ & 18 & $78^{\mathrm{cd}}$ & 2.7 & $3.7^{\mathrm{ab}}$ & 16.06 \\
\hline \multirow[t]{6}{*}{ Kekeba } & Mancozeb & $44.8^{\mathrm{abcd}}$ & 4.3 & $76.2^{\mathrm{ab}}$ & 0.6 & $4.96^{\mathrm{a}}$ & 1.8 & $37^{\text {bcdefg }}$ & 12 & $79^{\text {abcd }}$ & 1.8 & $4.3^{\mathrm{a}}$ & 2.06 \\
\hline & Tilt & $46.8^{\mathrm{a}}$ & 0 & $76.7^{\mathrm{ab}}$ & 0 & $4.9^{\mathrm{a}}$ & 3.9 & $42^{\mathrm{a}}$ & 0 & $80.57^{\mathrm{a}}$ & 0 & $4.36^{\mathrm{a}}$ & 0 \\
\hline & MMTT & $42.3^{\text {bcdef }}$ & 9.7 & $76.3^{\mathrm{ab}}$ & 0.5 & $4.5^{\mathrm{ab}}$ & 11 & $37^{\text {bcdefg }}$ & 11 & $79.9^{\mathrm{ab}}$ & 0.79 & $3.8^{\mathrm{ab}}$ & 12.16 \\
\hline & TTMM & $45.6^{\mathrm{ab}}$ & 2.6 & $76.5^{\mathrm{ab}}$ & 0.2 & $4.79^{\mathrm{a}}$ & 5.2 & $40^{\mathrm{abcd}}$ & 4.1 & $79^{\mathrm{abcd}}$ & 1.74 & $3.86^{\mathrm{ab}}$ & 12.06 \\
\hline & MTMT & $45.3^{\mathrm{abc}}$ & 3.1 & $76.3^{\mathrm{ab}}$ & 0.5 & $5.05^{\mathrm{a}}$ & 0 & $36^{\mathrm{efg}}$ & 15 & $79^{\text {bcd }}$ & 1.99 & $4.33^{\mathrm{a}}$ & 0.69 \\
\hline & Control & $36.9^{\mathrm{gh}}$ & 18 & $66.9^{c}$ & 11 & $3.12^{\mathrm{e}}$ & 34 & $34.8^{\mathrm{fg}}$ & 16 & $78^{d}$ & 1.9 & $2.9^{\mathrm{b}}$ & 39.3 \\
\hline \multirow[t]{8}{*}{ Madawalabu } & Mancozeb & $41.6^{\text {bcdef }}$ & 7.4 & $75.1^{\mathrm{ab}}$ & 0 & $4.3^{\mathrm{abcd}}$ & 8.7 & $37^{\text {bcdefg }}$ & 10 & $78^{\mathrm{cd}}$ & 1.8 & $4.47^{\mathrm{a}}$ & 6.5 \\
\hline & Tilt & $42.8^{\text {abcdef }}$ & 4.7 & $74.6^{\mathrm{ab}}$ & 0.6 & $4.5^{\mathrm{ab}}$ & 5.1 & $41^{\mathrm{ab}}$ & 0.6 & $79^{\mathrm{abcd}}$ & 0 & $4.78^{\mathrm{a}}$ & 0 \\
\hline & MMTT & $44.9^{\mathrm{abcd}}$ & 0 & $74.5^{\mathrm{ab}}$ & 0.7 & $4.7^{\mathrm{ab}}$ & 0 & $39.87^{\text {abcde }}$ & 3.5 & $78^{\mathrm{cd}}$ & 1.3 & $4.09^{\mathrm{ab}}$ & 14.4 \\
\hline & TTMM & $44.4^{\text {abcde }}$ & 1.2 & $73.7^{\mathrm{ab}}$ & 1.8 & $4.2^{\mathrm{abcde}}$ & 12 & $41.3^{\mathrm{a}}$ & 0 & $79^{\text {bcd }}$ & 0.2 & $4.33^{\mathrm{a}}$ & 9.4 \\
\hline & MTMT & $42.27^{\text {bcdef }}$ & 5.9 & $73.4^{\mathrm{ab}}$ & 2.2 & $3.6^{\text {bcde }}$ & 24 & & & & 0.9 & & 13 \\
\hline & & & & & & & & $37^{\text {cdefg }}$ & 11 & $79^{\mathrm{bcd}}$ & & $4.16^{\mathrm{ab}}$ & \\
\hline & Mean & 42.06 & & 74.95 & & 4.24 & & 38.2 & & 78.9 & & 4.2 & \\
\hline & $\mathrm{CV}$ & 5.18 & & 3.29 & & 14.98 & & 5.89 & & 0.96 & & 14.9 & \\
\hline
\end{tabular}

MMTT=Mancozeb-Mancozeb-Tilt-Tilt, TTMM=Tilt-Tilt-Mancozeb-Mancozeb, MTMT= Mancozeb-Tilt Mancozeb-Tilt, $\mathrm{CV}=$ Coefficient of Variation, $\mathrm{TKW}=$ Thousand kernel weight, HLW= Hectoliter weight, YLD= Yield, RTL $=$ Relative Thousand kernel weight loss, RHL $=$ Relative Hectolitter weight loss, $\mathrm{RYL}=$ Relative Yield loss, 
Yield and Yield Components Recovery

The highest yield recovery over unsprayed plot, 55\% and 65\%, were recorded from Kekeba variety sprayed with MTMT at Holeta and Tilt sprayed Madawalabu variety at Kulumsa (Table 3). These results were higher than those obtained from the work of Tari et al. (2009), which resulted in up to 42\% yield loss control by applying foliar fungicides to winter wheat. In general, on all varieties, STB developed after growth stage of Z70 (kernel and milk development stage) was found to be important for grain yield loss. So managing the disease before reaching this growth stage might provide reasonable recovery of yield. This might be related with protecting the top three leaves, especially flag leaf of the crop that contributes most to the grain yield (Vrapi et al., 2009).

\section{Correlation between Disease Parameters, Yield and Yield Components}

At both locations correlations among disease parameters were positive and highly significant $(\mathrm{p} \leq 0.01)$, suggesting the possibility of using any of the parameters for STB assessment. However, cautions should be taken as each parameter is worth considering having a complete understanding of disease intensity. STB severity, incidence and AUDPC also showed highly significant and negative correlations with grain yield, spike length, plant height, and weight of kernel per spike, kernel number per spike, thousand seed weight and hectoliter weight (Tables 4 \& 5). The highest value of correlation coefficient indicated strong relationships between and within disease parameters. Forrer and Zadoks (1983) also observed that the greatest risk to wheat crop occurs, when conducive environmental factors favor spore dispersal during and shortly after flag leaf emergence, and the crop losses have been related to total leaf area infected including necrotic lesions and chlorotic flakes.

Table 3. Effect of fungicide Spray Schedules on yield and yield components Recovery(\%) .

\begin{tabular}{|c|c|c|c|c|c|c|c|c|c|c|c|c|c|}
\hline \multirow{2}{*}{$\begin{array}{l}\text { Treatments } \\
\text { Variety }\end{array}$} & \multicolumn{6}{|c|}{ Holeta } & \multicolumn{7}{|c|}{ Kulumsa } \\
\hline & Fungicide & Yield & YIOUP & TKW & $\begin{array}{l}\text { TKW } \\
\text { IOUP }\end{array}$ & HLW & $\begin{array}{l}\text { HLW } \\
\text { IOUP }\end{array}$ & Yield & YIOUP & TKW & $\begin{array}{l}\text { TKW } \\
\text { IOUP }\end{array}$ & HLW & $\begin{array}{l}\text { HLW } \\
\text { IOUP }\end{array}$ \\
\hline \multirow[t]{4}{*}{ Alidoro } & Control & $3.2^{\mathrm{de}}$ & 0 & $39^{\mathrm{fg}}$ & 0 & $75^{\mathrm{ab}}$ & 0 & $3.7^{\mathrm{ab}}$ & 0 & $37^{\operatorname{defg}}$ & 0 & $78.5^{\text {bcd }}$ & 0 \\
\hline & Tilt & $4.9^{\mathrm{abcd}}$ & 54 & $41^{\text {cdef }}$ & 5.3 & $77^{a}$ & 3.1 & $4.7^{\mathrm{a}}$ & 26 & $39^{\text {abcdefg }}$ & 6.4 & $79^{\mathrm{abcd}}$ & 0.9 \\
\hline & MMTT & $4.3^{\mathrm{abc}}$ & 36 & $40^{\text {efg }}$ & 2.7 & $76^{\mathrm{ab}}$ & 2.4 & $4.4^{\mathrm{a}}$ & 17 & $39^{\text {abcdefg }}$ & 5.5 & $79^{\mathrm{abc}}$ & 1.1 \\
\hline & TTMM & $4.5^{\mathrm{ab}}$ & 42 & $41^{\text {defg }}$ & 4.0 & $77^{\mathrm{a}}$ & 3.1 & $4.6^{\mathrm{a}}$ & 23 & $37^{\text {bcdefg }}$ & 0.9 & $78.9^{\text {bcd }}$ & 0.5 \\
\hline \multirow{5}{*}{ Kekeba } & Mancoze & $4.96^{\mathrm{a}}$ & 52 & $45^{\text {abcd }}$ & 27 & $76^{\mathrm{ab}}$ & 4.8 & $4.3^{\mathrm{a}}$ & 17 & $37^{\text {bcdefg }}$ & 6.9 & $79^{\mathrm{abcd}}$ & 0.9 \\
\hline & Tilt & $4.9^{\mathrm{ab}}$ & 49 & $46.8^{\mathrm{a}}$ & 33 & $77^{\mathrm{ab}}$ & 5.5 & $4.4^{\mathrm{a}}$ & 19 & $42^{\mathrm{a}}$ & 21 & $80.6^{\mathrm{a}}$ & 2.8 \\
\hline & MMTT & $4.5^{\mathrm{abcd}}$ & 38 & $42^{\text {bcdef }}$ & 20 & $76^{\mathrm{ab}}$ & 4.9 & $3.8^{\mathrm{ab}}$ & 5 & $37^{\text {bcdefg }}$ & 7.3 & $79.9^{\mathrm{ab}}$ & 1.9 \\
\hline & TTMM & $4.8^{\mathrm{abc}}$ & 47 & $45.6^{\mathrm{ab}}$ & 30 & $77^{\mathrm{ab}}$ & 5.2 & $3.9^{\mathrm{ab}}$ & 6 & $40^{\text {abcd }}$ & 16 & $79^{\mathrm{abcd}}$ & 1.0 \\
\hline & MTMT & $5.1^{\mathrm{a}}$ & 55 & $45^{\mathrm{abc}}$ & 29 & $76^{\mathrm{ab}}$ & 4.9 & $4.3^{\mathrm{a}}$ & 18 & $35.6^{\mathrm{efg}}$ & 2.7 & $79^{\text {bcd }}$ & 0.7 \\
\hline \multirow{4}{*}{ Madawalab } & TTMM & $4.2^{\mathrm{abcd}}$ & 33 & $44^{\mathrm{abcde}}$ & 20 & $74^{\mathrm{ab}}$ & 10 & $4.3^{\mathrm{a}}$ & 49 & $41.3^{\mathrm{a}}$ & 19 & $79^{\text {bcd }}$ & 1.7 \\
\hline & MTMT & $3.6^{\text {bcde }}$ & 15 & $42^{\text {bcdef }}$ & 15 & $73^{\mathrm{ab}}$ & 9.7 & $4.2^{\mathrm{ab}}$ & 44 & $37^{\text {cdefg }}$ & 6 & $79^{\text {bcd }}$ & 0.9 \\
\hline & Mean & 4.24 & & 42.06 & & 74.9 & & 4.2 & & 38.16 & & 78.9 & \\
\hline & $\mathrm{CV}$ & 14.98 & & 5.18 & & 3.29 & & 43.5 & & 5.89 & & 0.96 & \\
\hline
\end{tabular}

Table 4. Correlation Coefficient among Disease Parameters, Yield and Yield Components at Holetta.

\begin{tabular}{|c|c|c|c|c|c|c|c|c|c|c|}
\hline & PDI & PDS & AUDPC & SL & $\mathrm{PH}$ & NKPS & YLD & TKW & HLW & ADS \\
\hline PDI & & & & & & & & & & \\
\hline PDS & $0.69 * *$ & & & & & & & & & \\
\hline AUDPC & $0.98 * *$ & $0.68 * *$ & & & & & & & & \\
\hline $\mathrm{SL}$ & $-0.59 * *$ & $-0.47 * *$ & $-0.59 * *$ & & & & & & & \\
\hline $\mathrm{PH}$ & $-0.47 * *$ & $-0.53^{* *}$ & $-0.47 * *$ & $0.72 * *$ & & & & & & \\
\hline NKPS & $-0.29 *$ & $-0.09^{\text {ns }}$ & $-0.29^{*}$ & $0.27 *$ & $0.26^{\mathrm{ns}}$ & & & & & \\
\hline YLD & $-0.43 * *$ & $-0.21^{\mathrm{ns}}$ & $-0.40 * *$ & $0.18^{\mathrm{ns}}$ & $0.23^{\mathrm{ns}}$ & $0.07^{\mathrm{ns}}$ & & & & \\
\hline TKW & $-0.33 * *$ & $-0.11^{\mathrm{ns}}$ & $-0.31^{*}$ & $-0.20^{\mathrm{ns}}$ & $-0.24^{\mathrm{ns}}$ & $-0.09^{\mathrm{ns}}$ & $0.53 * *$ & & & \\
\hline HLW & $-0.43^{* *}$ & $-0.19^{\mathrm{ns}}$ & $-0.39 * *$ & $0.12^{\mathrm{ns}}$ & $0.11^{\mathrm{ns}}$ & $0.03^{\mathrm{ns}}$ & $0.63 * *$ & $0.55^{* *}$ & & \\
\hline $\mathrm{ADS}$ & $0.98 * *$ & $0.67 * *$ & $0.99 * *$ & $-0.59 * *$ & $-0.46 * *$ & $-0.29 *$ & $-0.41 * *$ & $-0.31 *$ & $-0.39^{* *}$ & \\
\hline
\end{tabular}

$\mathrm{PDI}=$ Percent disease incidence, $\mathrm{PDS}=$ Percent disease severity, AUDPC $=$ Area under disease progress curve, $\mathrm{SL}=$ Spike length, $\mathrm{PH}=$ Plant height, NKPS = Number of kernels per spike, YLD $=$ Yield(t/ha), $\mathrm{TKW}=\mathrm{Thousand}$ kernel weight, HLW= Hecto litter weight and MDS= Mean disease severity.*:refers to mean square values significant at $\alpha=0.05, * *$ : refers to mean square values significant at $\alpha=0.01$, ns: refers to mean square values not significant at $\alpha=0.05$ 
Table 5. Correlation Coefficient among disease parameters, Yield and Yield Components at Kulumsa.

\begin{tabular}{|c|c|c|c|c|c|c|c|c|c|c|}
\hline & PDI & PDS & AUDPC & SL & $\mathrm{PH}$ & NKPS & YLD & TKW & HLW & ADS \\
\hline \multicolumn{11}{|l|}{ PDI } \\
\hline PDS & $0.88^{* *}$ & & & & & & & & & \\
\hline AUDPC & $0.97 * *$ & $0.92 * *$ & & & & & & & & \\
\hline SL & $-0.56 * *$ & $-0.42 * *$ & $-0.53 * *$ & & & & & & & \\
\hline $\mathrm{PH}$ & $-0.40 * *$ & $-0.29^{*}$ & $-0.42 * *$ & $0.74 * *$ & & & & & & \\
\hline NKPS & $-0.73 * *$ & $-0.57 * *$ & $-0.65^{* *}$ & $0.49 * *$ & $0.37^{* *}$ & & & & & \\
\hline YLD & $-0.29 *$ & $-0.32 *$ & $-0.31 *$ & $0.23^{\mathrm{ns}}$ & $0.29 *$ & $0.06^{\mathrm{ns}}$ & & & & \\
\hline TKW & $-0.17^{\mathrm{ns}}$ & $-0.26^{\mathrm{ns}}$ & $-0.21^{\mathrm{ns}}$ & $0.15^{\mathrm{ns}}$ & $-0.08^{\mathrm{ns}}$ & $0.01^{\mathrm{ns}}$ & $0.01^{\mathrm{ns}}$ & & & \\
\hline HLW & $-0.24^{\mathrm{ns}}$ & $-0.18^{\mathrm{ns}}$ & $-0.22^{\mathrm{ns}}$ & $-0.20^{\mathrm{ns}}$ & $-0.28^{*}$ & $0.18^{\mathrm{ns}}$ & $0.18^{\mathrm{ns}}$ & $0.30^{*}$ & & \\
\hline ADS & $0.97 * *$ & $0.92 * *$ & $0.99 * *$ & $-0.56 * *$ & $-0.45 * *$ & $-0.31 *$ & $-0.31 *$ & $-0.19^{n s}$ & $-0.19^{n s}$ & \\
\hline
\end{tabular}

$\mathrm{PDI}=$ Percent disease incidence, $\mathrm{PDS}=$ Percent disease severity, $\mathrm{AUDPC}=$ Area under disease progress curve, $\mathrm{SL}=$ Spike length, $\mathrm{PH}=$ Plant height, NKPS $=$ Number of kernels per spike, YLD $=$ Yield(t/ha), TKW= Thousand kernel weight, HLW= Hecto litter weight and MDS= Mean disease severity. ${ }^{*}$ :refers to mean square values significant at $\alpha=0.05, * *$ : refers to mean square values significant at $\alpha=0.01$, ns: refers to mean square values not significant at $\alpha=0.05$

\section{Conclusion and recommendation}

A field experiment was conducted at Holetta and Kulumsa in 2016 main cropping season to determine the effect of STB disease on yield and yield components loss and recovery of bread wheat varieties. At Holetta, maximum relative grain yield loss was $36 \%$, and this was recorded on unsprayed plots of the variety Kekeba whereas at Kulumsa, maximum relative grain yield loss was $39 \%$ on unsprayed plots of the variety Madawalabu. The highest yield recovery over unsprayed plot, $55 \%$ and $65 \%$, were recorded from Kekeba variety sprayed with MTMT at Holeta and Tilt sprayed Madawalabu variety at Kulumsa. STB resulted in significant yield loss of bread wheat varieties, when left unchecked. STB severity, incidence and AUDPC also showed highly significant and negative correlations with grain yield and yield components.

\section{Acknowledgments}

I would like to thank the Almighty God and St. Marry for making all things possible. I would like to express my sincere thanks to all concerned peoples who are positive thinkers. My special thanks goes to the staff of Holeta and Kulumsa Agricultural Research Centers to support me financially and morally. Thanks to God and the mother of my Lord for making all ups and down possible.

\section{REFERENCES}

Ayele A and Muche G (2019). Yield Loss Assessment in Bread Wheat Varieties Caused by Yellow Rust (Puccinia striiformis f. sp. tritici) in Arsi Highlands of South Eastern Ethiopia. American Journal of BioScience 7(6): 104-112.

Beyene AT, Derera J, Sibiya J (2016). Gene action determining grain yield and chocolate spot (Botrytis fabae) resistance in faba bean. Euphytica 207: 293-304.

Blandford D and Hassapoyannes K (2018). The role of agriculture in global GHG mitigation.

Birhan Abdulkadir (2011). KARC Stations distribution and website description.

Campbell CL and Madden LV (1990). Introduction to plant disease epidemiology. John W. \& Sons,New York City.pp.386-427.

Central Statistics Agency (CSA) (2017). Agricultural Sample Survey. Report on Area and Production of Major Crops. Statistical Bulletin 584,Volume1,Addis Ababa,Ethiopia.

Endale Hailu and Getaneh Woldeab (2015). Survey of Rust and Septoria Leaf Blotch Diseases of Wheat in Central Ethiopia and Virulence Diversity of Stem Rust Puccinia graminis f. sp. tritici. Advanced Crop Science Technology 3(2):2-5.

Eyal Z (1987). The Septoria diseases of wheat: concepts and methods of disease management. pp.52.

Food and Agricultural Organization (FAO) (2017). Crop Prospects and Food situation: Global Cereal Production brief.News room available at :http://www.fao.org/newsroom/en/news/23Dec,2017/1000805/index.html.

Forrer HR and Zadoks JC (1983). Yield reduction in wheat in relation to leaf necrosis caused by Septoria tritici. Netherlands Journal of Plant Pathology 89(3):87-98.

McDonald BA and Mundt CC (2016). How knowledge of pathogen population biology informs management of Septoria tritici blotch. Phytopathology 106(9):948-955.

Miedaner T, Schmitt AK, Klocke B, Schmiedchen B, Wilde P, Spieß H, Szabo L, Koch S and Flath K (2016). Analyzing genetic diversity for virulence and resistance phenotypes in populations of stem rust (Puccinia graminis f. sp. secalis) and winter rye (Secale cereale). Phytopathology 106(11):1335-1343. 
Negasa D and Chauhan DK (2016). Variability, Heritability and Genetic Advances in Wheat (Triticum aestivum L.) Breeding lines grown at Horro Guduru Wollega Zone, Western Ethiopia. International Journal of Advanced Scientific Research and Management 1(1):24-28.

Ponomarenko A, Goodwin SB and Kema GHJ (2011). Septoria tritici blotch (STB) of wheat. Plant Health .Department of Botany and Plant Pathology, Purdue University.pp.1-10.

Randhawa MS, Bhavani S, Singh PK, Huerta-Espino J and Singh R.P (2019). Disease Resistance in Wheat: Present Status and Future Prospects. In Disease Resistance in Crop Plants .Springer, Cham. pp. 61-81.

Saari EE and Prescott JM (1975). Scale for appraising the foliar intensity of wheat diseases. Plant Disease Reporter 59:377-380.

Said A and Hussein T (2016). Epidemics of Septoria tritici blotch and its development overtime on bread wheat in Haddiya-Kambata area of Southern Ethiopia. Journal of Biology, Agriculture and Health Care 6(1) :47-57.

Serfling A, Kopahnke D, Habekuss A, Novakazi F and Ordon F (2017). Wheat diseases: an overview. Achieving sustainable cultivation of wheat.

SAS(Statistical Analysis System) (2014). Statistical Analysis System SAS/STAT user's guide Version9.3. Carry,North Carolina,SAS Institute Inc. USA.

Sharma R and Duveiller E (2007). Advancement toward new Spot Blotch resistant wheat in South Asia. Crop Science 47:961-968.

Singh RP, Singh PK, Rutkoski J, Hodson DP, He X, Jørgensen LN, Hovmøller MS and Huerta-Espino J (2016). Disease impact on wheat yield potential and prospects of genetic control. Annual review of phytopathology 54:303-322.

Tadesse Y, Chala A and Kassa B (2018). Survey of Septoria Tritici Blotch (Septoria Tritici) of Bread Wheat (Triticum aestivum L.) in the Central Highlands of Ethiopia. American Journal of Bioscience and Bioengineering 6(5):36-41.

Takele A, Lencho A, Getaneh WA, Hailu E and Kassa B (2015). Status of wheat Septoria leaf blotch (Septaria tritici Roberge in Desmaz) in south west and Western Shewa zones of Oromiya regional state, Ethiopia. Research in Plant Sciences 3(3) :43-48.

Tari DB, Gazanchian A, Pirdashti HA and Nasiri M (2009). Flag leaf morpho-physiological response to different agronomical treatments in a promising line of rice(Oryza sativa L.). American Euroasean Journal of Agriculture and Environmental Science 5: 403-408.

Teklay Abebe, Muez Mehari and Muruts Legesse (2015). Field Response of wheat genotypes to Septoria tritici blotch in Tigray, Ethiopia. Journal of Natural Sciences Research 5(1):146-152.

Vrapi H, Belul G, Foto K, Halit S and Thanas R (2012). The relationship between diseases index of septoria leaf blotch, leaf rust and yield losses in bread wheat cultivar in Albania. Journal of Environmental Science and Engineering 1:957-965. 